\title{
A theorem of Cobham for non-primitive substitutions
}

\author{
by \\ FABien Durand (Amiens)
}

1. Introduction. Given a subset $E$ of $\mathbb{N}=\{0,1,2, \ldots\}$ can we find an elementary algorithm (i.e., a finite state automaton) which accepts the elements of $E$ and rejects those that do not belong to $E$ ? In 1969 A. Cobham showed that the existence of such an algorithm deeply depends on the numeration base. He stated [Co1]: Let $p$ and $q$ be two multiplicatively independent integers (i.e., $p^{k} \neq q^{l}$ for all integers $k, l>0$ ) greater than or equal to 2 . Let $E \subset \mathbb{N}$. The set $E$ is both p-recognizable and q-recognizable if and only if $E$ is a finite union of arithmetic progressions. This is now called the theorem of Cobham. We recall that a set $E \subset \mathbb{N}$ is p-recognizable for some integer $p \geq 2$ if the language consisting of the expansions in base $p$ of the elements of $E$ is recognizable by a finite state automaton (see [Ei]).

In 1972 Cobham [Co2] gave another partial answer to this question showing that not all sets are $p$-recognizable. He gave the following characterization: The set $E \subset \mathbb{N}$ is p-recognizable for some integer $p \geq 2$ if and only if the characteristic sequence $\left(x_{n} ; n \in \mathbb{N}\right)$ of $E\left(x_{n}=1\right.$ if $n \in E$ and 0 otherwise) is generated by a substitution of length $p$, where "generated by a substitution of length $p$ " means that it is the image by a letter-to-letter morphism of a fixed point of a substitution of length $p$.

We remark that $E$ is a finite union of arithmetic progressions if and only if its characteristic sequence is ultimately periodic. Consequently, the theorem of Cobham can be formulated as follows (this is an equivalent statement): Let $p$ and $q$ be two multiplicatively independent integers greater than or equal to 2. Let $A$ be a finite alphabet and $\mathrm{x} \in A^{\mathbb{N}}$. The sequence $\mathrm{x}$ is generated by both a substitution of length $p$ and a substitution of length $q$ if and only if $\mathrm{x}$ is ultimately periodic.

To a substitution $\sigma$ is associated an integer square matrix $M \neq 0$ which has non-negative entries. It is known (see $[\mathrm{LM}]$ for instance) that such a matrix has a real eigenvalue $\alpha$ which is greater than or equal to the modulus

2000 Mathematics Subject Classification: Primary 11B85; Secondary 68R15.

Key words and phrases: substitutions, substitutive sequences, theorem of Cobham. 
of all other eigenvalues. It is usually called the dominant eigenvalue of $M$. Let $\mathcal{S}$ be a set of substitutions. If $\mathrm{x}$ is the image by a letter-to-letter morphism of a fixed point of $\sigma \in \mathcal{S}$ then we will say that $\mathrm{x}$ is $\alpha$-substitutive in $\mathcal{S}$. If $\mathcal{S}$ is the set of all substitutions we will say that $\mathrm{x}$ is $\alpha$-substitutive. An easy computation shows that if $\sigma$ is of length $p$ then $\alpha=p$. Furthermore if a sequence is generated by a substitution of length $p$ then it is $p$-substitutive. Note that the converse is not true. This suggests the following conjecture formulated by G. Hansel.

Conjecture. Let $\alpha$ and $\beta$ be two multiplicatively independent Perron numbers. Let $A$ be a finite alphabet. Let $\times$ be a sequence in $A^{\mathbb{N}}$. The following are equivalent:

(1) $\mathrm{x}$ is both $\alpha$-substitutive and $\beta$-substitutive;

(2) $\times$ is ultimately periodic.

In this paper we prove that (2) implies (1) and, what is the main result of this paper, that this conjecture holds for a very large set of substitutions containing all known cases; we call it $\mathcal{S}_{\text {good }}$. This set contains some non-primitive substitutions of non-constant length. More precisely for some sets $\mathcal{S}$ of substitutions, we prove

Theorem 1. Let $\alpha$ and $\beta$ be two multiplicatively independent Perron numbers. Let $A$ be a finite alphabet. A sequence $\mathrm{x} \in A^{\mathbb{N}}$ is $\alpha$-substitutive in $\mathcal{S}$ and $\beta$-substitutive in $\mathcal{S}$ if and only if it is ultimately periodic.

This result is true for $\mathcal{S}_{\text {const }}$, the family of substitutions with constant length (this is the theorem of Cobham), and for $\mathcal{S}_{\text {prim }}$, the family of primitive substitutions [Du2]. In [Fa] and [Du3] this result was proved for families of substitutions related to numeration systems. These families contain some non-primitive substitutions of non-constant length.

Much more results have been proved concerning generalizations of Cobham's theorem to non-standard numeration systems [BHMV1, BHMV2].

Most of the proofs of Cobham's type results are divided into two parts. In the first part it is proven that the set $E \subset \mathbb{N}$ is syndetic (the difference between two consecutive elements of $E$ is bounded), which corresponds to the fact that the letters of the characteristic sequence of $E$ appear with bounded gaps. In the second part the result is proven for such $E$. We will do the same.

In Section 2 we recall some results concerning the length of the words $\sigma^{n}(a)$ where $\sigma$ is a substitution on the alphabet $A$ and $a \in A$. These results have a key role in this paper. In Section 3 we prove that (2) implies (1). To prove the syndeticity of $E$ all proofs use the well known fact that, if $\alpha$ and $\beta$ are multiplicatively independent numbers strictly greater than 1 then the set $\left\{\alpha^{n} / \beta^{m} ; n, m \in \mathbb{Z}\right\}$ is dense in $\mathbb{R}^{+}$. Here we need more: the density in $\mathbb{R}^{+}$of the set $\left\{n^{d} \alpha^{n} /\left(m^{e} \beta^{m}\right) ; n, m \in \mathbb{Z}\right\}$, where $d$ and $e$ are non-negative integers. 
We prove this result in Section 4 because we did not find it in the literature. We prove in Section 5 that the letters with infinitely many occurrences in $\mathrm{x} \in A^{\mathbb{N}}$ appear with bounded gaps. This implies the same result for words. In the last section we restrict ourselves to $\mathcal{S}_{\text {good }}$, we recall some results of [Du3] and, using return words, we conclude that $\mathrm{x}$ is ultimately periodic. More precisely we prove that the conjecture is true for $\mathcal{S}_{\text {good }}$.

Words and sequences. An alphabet $A$ is a finite set of elements called letters. A word on $A$ is an element of the free monoid generated by $A$, denoted by $A^{*}$. Let $x=x_{0} x_{1} \ldots x_{n-1}$ (with $x_{i} \in A, 0 \leq i \leq n-1$ ) be a word, its length is $n$ and is denoted by $|x|$. The empty word is denoted by $\epsilon$, $|\epsilon|=0$. The set of non-empty words on $A$ is denoted by $A^{+}$. The elements of $A^{\mathbb{N}}$ are called sequences. If $\mathrm{x}=\mathrm{x}_{0} \mathrm{x}_{1} \ldots$ is a sequence (with $\mathrm{x}_{i} \in A, i \in \mathbb{N}$ ), and $I=[k, l]$ an interval of $\mathbb{N}$ we set $\mathrm{x}_{I}=\mathrm{x}_{k} \mathrm{x}_{k+1} \ldots \mathrm{x}_{l}$ and we say that $\mathrm{x}_{I}$ is a factor of $\mathrm{x}$. If $k=0$, we say that $\mathrm{x}_{I}$ is a prefix of $\mathrm{x}$. The set of factors of length $n$ of $\mathrm{x}$ is denoted by $L_{n}(\mathrm{x})$, and the set of factors of $\mathrm{x}$, or language of $\mathrm{x}$, by $L(\mathrm{x})$. The occurrences in $\mathrm{x}$ of a word $u$ are the integers $i$ such that $\mathrm{x}_{[i, i+|u|-1]}=u$. When $\mathrm{x}$ is a word, we use the same terminology with similar definitions.

The sequence $\mathrm{x}$ is ultimately periodic if there exist a word $u$ and a nonempty word $v$ such that $\mathrm{x}=u v^{\omega}$, where $v^{\omega}=v v v \ldots$ Otherwise we say that $\mathrm{x}$ is non-periodic. It is periodic if $u$ is the empty word. A sequence $\mathrm{x}$ is uniformly recurrent if for each factor $u$ the greatest difference of two successive occurrences of $u$ is bounded.

Morphisms and matrices. Let $A$ and $B$ be two alphabets. A morphism $\tau$ is a map from $A$ to $B^{*}$. Such a map induces by concatenation a morphism from $A^{*}$ to $B^{*}$. If $\tau(A)$ is included in $B^{+}$, it induces a map from $A^{\mathbb{N}}$ to $B^{\mathbb{N}}$. These two maps are also called $\tau$.

To a morphism $\tau$, from $A$ to $B^{*}$, there is naturally associated the matrix $M_{\tau}=\left(m_{i, j}\right)_{i \in B, j \in A}$ where $m_{i, j}$ is the number of occurrences of $i$ in the word $\tau(j)$. Let $M$ be a square matrix. A dominant eigenvalue of $M$ is an eigenvalue $r$ such that no other eigenvalue exceeds it in modulus. A square matrix is called primitive if it has a power with positive coefficients. In this case the dominant eigenvalue is unique, positive and it is a simple root of the characteristic polynomial. This is Perron's theorem.

A real number is a Perron number if it is an algebraic integer that strictly dominates all its other algebraic conjugates. The following result is well known (see $[\mathrm{LM}]$ for instance).

TheOREM 2. Let $\lambda$ be a real number. Then

(1) $\lambda$ is a Perron number if and only if it is the dominant eigenvalue of a primitive non-negative integral matrix. 
(2) $\lambda$ is the spectral radius of a non-negative integral matrix if and only if $\lambda^{p}$ is a Perron number for some positive integer $p$.

Substitutions and substitutive sequences. In this paper a substitution is a morphism $\tau: A \rightarrow A^{*}$ such that for all letters of $A$ we have $\lim _{n \rightarrow \infty}\left|\tau^{n}(a)\right|$ $=\infty$. Whenever the matrix associated to $\tau$ is primitive we say that $\tau$ is a primitive substitution.

A fixed point of $\tau$ is a sequence $\mathrm{x}=\left(\mathrm{x}_{n} ; n \in \mathbb{N}\right)$ such that $\tau(\mathrm{x})=\mathrm{x}$. We say it is a proper fixed point if all letters of $A$ have an occurrence in $\mathrm{x}$. We remark that all proper fixed points of $\tau$ have the same language.

ExAMPLE. The substitution $\tau$ defined by $\tau(a)=a a a b, \tau(b)=b c$ and $\tau(c)=b$ has two fixed points; one starts with $a$ and is proper and the other stars with $b$ and is not proper.

If $\tau$ is a primitive substitution then all its fixed points are proper and uniformly recurrent (for details see [Qu] for example).

Let $B$ be another alphabet. We say that a morphism $\phi$ from $A$ to $B^{*}$ is a letter-to-letter morphism when $\phi(A)$ is a subset of $B$. Let $\mathcal{S}$ be a set of substitutions and suppose that $\tau$ belongs to $\mathcal{S}$. Then the sequence $\phi(\mathrm{x})$ is called substitutive in $\mathcal{S}$. We say $\phi(\mathrm{x})$ is substitutive (resp. primitive substitutive) if $\mathcal{S}$ is the set of all substitutions (resp. the set of primitive substitutions). If $\mathrm{x}$ is a proper fixed point of $\tau$ and $\theta$ is the dominant eigenvalue of $\tau \in \mathcal{S}$ (i.e., the dominant eigenvalue of the matrix associated to $\tau$ ) then $\phi(x)$ is called $\theta$-substitutive in $\mathcal{S}$; and we say $\theta$-substitutive (resp. primitive substitutive) if $\mathcal{S}$ is the set of all substitutions (resp. the set of primitive substitutions).

We point out that in the last example the fixed point y of $\tau$ starting with the letter $b$ is also the fixed point of the substitution $\sigma$ defined by $\sigma(b)=b c$ and $\sigma(c)=b$. Moreover the dominant eigenvalue of $\tau$ is 3 and the dominant eigenvalue of $\sigma$ is $(1+\sqrt{5}) / 2$. Hence in the definition of " $\theta$-substitutive" it is very important for $x$ to be a proper fixed point, otherwise the conjecture presented in the introduction would not be true.

Clearly, if $\phi(\mathbf{x})$ is $\theta$-substitutive then it is $\theta^{p}$-substitutive for all $p \in \mathbb{N}$. Consequently, from Theorem 2 we can always suppose $\theta$ is a Perron number. We define

$$
L(\tau)=\left\{\tau^{n}(a)_{[i, j]} ; i, j \in \mathbb{N}, i \leq j, n \in \mathbb{N}, a \in A\right\} .
$$

Let $\mathrm{x}$ be a fixed point of $\tau$. Then $L(\tau)=L(\mathrm{x})$ if and only if $\mathrm{x}$ is proper. If $\tau$ is primitive then all its fixed points $\mathrm{x}$ have the same language $L=L(\tau)$.

2. Some preliminary lemmata. This section and the first case of the proof of Proposition 13 is prompted by the ideas in [Ha]. In this section $\sigma$ will denote a substitution defined on the finite alphabet $A$, x one of its fixed points and $\Theta$ its dominant eigenvalue. 
Lemma 3. There exists a unique partition $A_{1}, \ldots, A_{l}$ of $A$ such that for all $1 \leq i \leq l$ and all $a \in A_{i}$,

$$
\lim _{n \rightarrow \infty} \frac{\left|\sigma^{n}(a)\right|}{c(a) n^{d(a)} \theta(a)^{n}}=1
$$

where $\theta(a)$ is the dominant eigenvalue of $M$ restricted to $A_{i}, d(a)$ its Jordan order and $c(a) \in \mathbb{R}$.

Proof. See Theorem II.10.2 in [SS].

For all $a \in A$ we will call the couple $(d(a), \theta(a))$ the growth type of $a$. If $(d, \alpha)$ and $(e, \beta)$ are two growth types we say that $(d, \alpha)$ is less than $(e, \beta)$ (written $(d, \alpha)<(e, \beta))$ whenever either $\alpha<\beta$, or $\alpha=\beta$ and $d<e$. Consequently, if the growth type of $a \in A$ is less than the growth type of $b \in A$ then $\lim _{n \rightarrow \infty}\left|\sigma^{n}(a)\right| /\left|\sigma^{n}(b)\right|=0$. If the growth type of $a \in A$ is $(i, \theta)$ then there exists a letter $b$ with growth type $(i, \theta)$ having an occurrence in $\sigma(a)$.

We have $\Theta=\max \{\theta(a) ; a \in A\}$. We set $D=\max \{d(a) ; \theta(a)=\Theta$, $a \in A\}$ and $A_{\max }=\{a \in A ; \theta(a)=\Theta, d(a)=D\}$. We will say that the letters of $A_{\max }$ are of maximal growth and that $(D, \Theta)$ is the growth type of $\sigma$.

For all letters $a \in A$, as $\lim _{n \rightarrow \infty}\left|\sigma^{n}(a)\right|=\infty$, we have either $\theta(a)>1$, or $\theta(a)=1$ and $d(a)>0$. Hence Lemma 3 implies that there is no letter with growth type $(0,1)$. An important consequence of the following lemma is that in fact $\theta(a)>1$ for all $a \in A$.

LEMma 4. If $(d, \theta)$ is the growth type of some letter then for all $i$ belonging to $\{0, \ldots, d\}$ there exists a letter of growth type $(i, \theta)$ which appears infinitely often in $\mathrm{x}$.

Proof. See Lemma III.7.10 in [SS].

We define

$$
\lambda_{\sigma}: A^{*} \rightarrow \mathbb{R}, \quad u_{0} \ldots u_{n-1} \mapsto \sum_{i=0}^{n-1} c\left(u_{i}\right) \mathbf{1}_{A_{\max }}\left(u_{i}\right) .
$$

From Lemma 3 we deduce the following lemma.

Lemma 5. For all $u \in A^{*}$ we have $\lim _{n \rightarrow \infty}\left|\sigma^{n}(u)\right| /\left(n^{D} \Theta^{n}\right)=\lambda_{\sigma}(u)$.

We say that $u \in A^{*}$ is of maximal growth if $\lambda_{\sigma}(u) \neq 0$.

Lemma 6. Suppose $a \in A$ has infinitely many occurrences in $\mathrm{x}$. There exist a positive integer $p$, a word $u \in A^{*}$ of maximal growth and $v, w \in A^{*}$ such that for all $n \in \mathbb{N}$ the word

$$
\sigma^{p n}(u) \sigma^{p(n-1)}(v) \sigma^{p(n-2)}(v) \ldots \sigma^{p}(v) v w a
$$

is a prefix of $\mathrm{x}$. Moreover,

$$
\lim _{n \rightarrow \infty} \frac{\left|\sigma^{p n}(u) \sigma^{p(n-1)}(v) \sigma^{p(n-2)}(v) \ldots \sigma^{p}(v) v w a\right|}{\lambda_{\sigma}(u)(p n)^{D} \Theta^{p n}+\lambda_{\sigma}(v) \sum_{k=0}^{n-1}(p k)^{D} \Theta^{p k}}=1 .
$$


Proof. Let $a \in A$ be a letter that has infinitely many occurrences in $\mathrm{x}$. We set $a_{0}=a$. There exists $a_{1} \in A$ which has infinitely many occurrences in $\mathrm{x}$ and such that $a_{0}$ has an occurrence in $\sigma\left(a_{1}\right)$. In this way we can construct a sequence $\left(a_{i} ; i \in \mathbb{N}\right)$ such that $a_{0}=a$ and $a_{i}$ occurs in $\sigma\left(a_{i+1}\right)$, for all $i \in \mathbb{N}$. There exist $i, j$ with $i<j$ such that $a_{i}=a_{j}=b$. It comes that $a$ occurs in $\sigma^{i}(b)$ and $b$ occurs in $\sigma^{j-i}(b)$. Hence there exist $u_{1}, u_{2}, v_{1}, v_{2} \in A^{*}$ such that $\sigma^{i}(b)=u_{1} a u_{2}$ and $\sigma^{j-i}(b)=v_{1} b v_{2}$. We set $p=j-i, v=\sigma^{i}\left(v_{1}\right)$ and $w=u_{1}$. There exists $u^{\prime}$ such that $u^{\prime} b$ is a prefix of $\mathbf{x}$. We remark that for all $n \in \mathbb{N}$ the word $\sigma^{n}\left(u^{\prime} b\right)$ is also a prefix of $\mathrm{x}$. We set $u=\sigma^{i}\left(u^{\prime}\right)$. We have $\sigma^{p}\left(u^{\prime} b\right)=\sigma^{p}\left(u^{\prime}\right) v_{1} b v_{2}$. Consequently, for all $n \in \mathbb{N}$,

$$
\sigma^{p n}\left(u^{\prime}\right) \sigma^{p(n-1)}\left(v_{1}\right) \sigma^{p(n-2)}\left(v_{1}\right) \ldots \sigma^{p}\left(v_{1}\right) v_{1} b
$$

is a prefix of $\sigma^{n p}\left(u^{\prime} b\right)$. Then

$$
\sigma^{p n}(u) \sigma^{p(n-1)}(v) \sigma^{p(n-2)}(v) \ldots \sigma^{p}(v) v w a
$$

is a prefix of $\sigma^{n p+i}\left(u^{\prime} b\right)$ and consequently of $\mathrm{x}$, for all $n \in \mathbb{N}$. The last part of the lemma follows from Lemma 5.

3. Assertion (2) implies assertion (1) in the Conjecture. In this section we prove the following proposition. It is the "easy" part of the Conjecture, namely assertion (2) implies assertion (1). The first part of the proof is an adaptation of the proof of Proposition 3.1 in [Du1] and the second part is inspired by the substitutions introduced in Sections V.4 and V.5 of [Qu].

Proposition 7. Let $\mathrm{x}$ be a sequence on a finite alphabet and $\alpha$ a Perron number. If $\mathrm{x}$ is periodic (resp. ultimately periodic) then it is $\alpha$-substitutive primitive (resp. $\alpha$-substitutive).

Proof. Let $\mathrm{x}$ be a periodic sequence with period $p$. Hence we can suppose that $A=\{1, \ldots, p\}$ and $\mathrm{x}=(1 \ldots p)^{\omega}$. Let $M$ be a primitive matrix whose dominant eigenvalue is $\alpha$ and $\sigma: B \rightarrow B^{*}$ a primitive substitution whose matrix is $M$. Let y be one of its fixed points. In what follows we construct, using $\sigma$, a new substitution $\tau$ with dominant eigenvalue $\alpha$, together with a fixed point $z=\tau(z)$, and a letter-to-letter morphism $\phi$ such that $\phi(z)=x$. We define the alphabet

$$
D=\{(b, i) ; b \in B, 1 \leq i \leq p\},
$$

the morphism $\psi: B \rightarrow D^{*}$ and the substitution $\tau: D \rightarrow D^{*}$ by

$$
\psi(b)=(b, 1) \ldots(b, p) \quad \text { and } \quad \tau((b, i))=(\psi(\sigma(b)))_{[(i-1)|\sigma(b)|, i|\sigma(b)|-1]}
$$

for all $(b, i) \in D$. The substitution $\tau$ is well defined because $|\psi(\sigma(b))|=$ $p|\sigma(b)|$. Moreover, these morphisms are such that $\tau \circ \psi=\psi \circ \sigma$. Hence the substitution $\tau$ is primitive. The sequence $\mathbf{z}=\psi(\mathrm{y})$ is a fixed point of $\tau$ and 
(using the Perron theorem and the fact that $M_{\tau} M_{\psi}=M_{\psi} M_{\sigma}$ ) its dominant eigenvalue is $\alpha$.

Let $\phi: D \rightarrow A$ be the letter-to-letter morphism defined by $\phi((b, i))=i$. It is easy to see that $\phi(z)=x$. It follows that $\mathrm{x}$ is $\alpha$-substitutive.

Suppose now that $x$ is ultimately periodic. Then there exist two nonempty words $u$ and $v$ such that $\mathrm{x}=u v^{\omega}$. From what precedes we know that there exist a substitution $\tau: D \rightarrow D^{*}$, a fixed point $\mathbf{z}=\tau(\mathrm{z})$ and a letterto-letter morphism $\phi: D \rightarrow A$ such that $\phi(z)=v^{\omega}$. Let $E^{\prime}=\left\{a_{1}, \ldots, a_{|u|}\right\}$ be an alphabet, with $|u|$ letters, disjoint from $D$ and consider the sequence $\mathrm{t}=a_{1} \ldots a_{|u|} \mathrm{z} \in\left(E^{\prime} \cup D\right)^{\mathbb{N}}=F^{\mathbb{N}}$. It suffices to prove that $\mathrm{t}$ is $\alpha$-substitutive. We extend $\tau$ to $F$ setting $\tau\left(a_{i}\right)=a_{i}$ for $1 \leq i \leq|u|$. Let $G$ be the alphabet of the words of length $|u|+1$ of $t$, that is to say,

$$
G=\left\{\left(\mathrm{t}_{n} \mathrm{t}_{n+1} \ldots \mathrm{t}_{n+|u|}\right) ; n \in \mathbb{N}\right\} \quad \text { where } \quad \mathrm{t}=\mathrm{t}_{0} \mathrm{t}_{1} \ldots
$$

The sequence $\overline{\mathrm{t}}=\left(\mathrm{t}_{0} \mathrm{t}_{1} \ldots \mathrm{t}_{|u|}\right)\left(\mathrm{t}_{1} \mathrm{t}_{2} \ldots \mathrm{t}_{|u|+1}\right) \ldots\left(\mathrm{t}_{n} \mathrm{t}_{n+1} \ldots \mathrm{t}_{n+|u|}\right) \ldots \in G^{\mathbb{N}}$ is a fixed point of the substitution $\zeta: G \rightarrow G^{*}$ that we define as follows. Let $\left(l_{0} l_{1} \ldots l_{|u|-1} a\right)$ be an element of $G$. Let $s_{0} s_{1} \ldots s_{|u|-1}$ be the suffix of length $|u|$ of the word $\tau\left(l_{0} l_{1} \ldots l_{|u|-1}\right)$.

If $|\tau(a)| \leq|u|$, we set

$$
\begin{aligned}
\zeta\left(\left(l_{0} l_{1} \ldots l_{|u|-1} a\right)\right) \\
\quad=\left(s_{[0,|u|-1]} \tau(a)_{0}\right)\left(s_{[1,|u|-1]} \tau(a)_{[0,1]}\right) \ldots\left(s_{[|\tau(a)|-1,|u|-1]} \tau(a)_{[0,|\tau(a)|-1]}\right),
\end{aligned}
$$

otherwise

$$
\begin{aligned}
& \zeta\left(\left(l_{0} l_{1} \ldots l_{|u|-1} a\right)\right) \\
& =\left(s_{[0,|u|-1]} \tau(a)_{0}\right) \ldots\left(s_{|u|-1} \tau(a)_{[0,|u|-1]}\right)\left(\tau(a)_{[0,|u|]}\right) \ldots\left(\tau(a)_{[|\tau(a)|-|u|-1,|\tau(a)|-1]}\right) .
\end{aligned}
$$

By induction we can prove that for all $n \in \mathbb{N}$ we have

$$
\begin{aligned}
& \zeta^{n}\left(\left(\mathrm{t}_{0} \mathrm{t}_{1} \ldots \mathrm{t}_{|u|}\right)\right) \\
& \quad=\left(\mathrm{t}_{0} \mathrm{t}_{1} \ldots \mathrm{t}_{|u|}\right)\left(\mathrm{t}_{1} \mathrm{t}_{2} \ldots \mathrm{t}_{|u|+1}\right) \ldots\left(\mathrm{t}_{\left|\tau^{n}\left(\mathrm{t}_{|u|}\right)\right|-1} \ldots \mathrm{t}_{\left|\tau^{n}\left(\mathrm{t}_{|u|}\right)\right|+|u|-1}\right) .
\end{aligned}
$$

Consequently, $\overline{\mathrm{t}}$ is a fixed point of $\zeta$ and $\varrho(\overline{\mathrm{t}})=\mathrm{t}$ where $\varrho: G \rightarrow F$ is defined by

$$
\varrho\left(\left(r_{0} r_{1} \ldots r_{|u|}\right)\right)=r_{0} .
$$

Moreover we remark that for all $n \in \mathbb{N}$ we have

$$
\left|\zeta^{n}\left(\left(r_{0} r_{1} \ldots r_{|u|}\right)\right)\right|=\left|\tau^{n}\left(r_{|u|}\right)\right| \text {. }
$$

From this and Lemma 3 it follows that for all $\left(r_{0} r_{1} \ldots r_{|u|}\right) \in D$ we have

$$
\lim _{n \rightarrow \infty} \frac{\left|\zeta^{n+1}\left(\left(r_{0} r_{1} \ldots r_{|u|}\right)\right)\right|}{\left|\zeta^{n}\left(\left(r_{0} r_{1} \ldots r_{|u|}\right)\right)\right|}=\alpha \text {. }
$$

Hence $\alpha$ is the dominant eigenvalue of $\zeta$ and $\mathrm{t}$ is $\alpha$-substitutive. 
EXAMPLE. Let $\mathrm{x}=(12)^{\omega}$ and $\alpha=(1+\sqrt{5}) / 2$. It is the dominant eigenvalue of the substitution $\sigma: A=\{a, b\} \rightarrow A^{*}$ given by $\sigma(a)=a b$ and $\sigma(b)=a$. We have $D=\{(a, 1),(a, 2),(b, 1),(b, 2)\}$ and the substitution $\tau: D \rightarrow D^{*}$ defined in the previous proof is given by

$$
\begin{aligned}
\tau((a, 1)) & =(a, 1)(a, 2), & & \tau((a, 2))=(b, 1)(b, 2), \\
\tau((b, 1)) & =(a, 1), & \tau((b, 2)) & =(a, 2) .
\end{aligned}
$$

EXAMPLE. Let $c$ be a letter and $\mathrm{x}=c(12)^{\omega}$. We adopt the notations of the previous example and for convenience we set $A=(a, 1), B=(a, 2)$, $C=(b, 1)$ and $D=(b, 2)$. The substitution $\zeta: G \rightarrow G^{*}$, where $G=$ $\{(c A),(A B),(B C),(C D),(D A),(B A)\}$, defined in the previous proof is given by

$$
\begin{aligned}
\zeta((c A)) & =((c A))((A B)), & \zeta((A B)) & =((B C))((C D)), \\
\zeta((B C)) & =((D A)), & \zeta((C D)) & =((A B)), \\
\zeta((D A)) & =((B A))((A B)), & \zeta((B A)) & =((D A))((A B)) .
\end{aligned}
$$

Let $\overline{\mathrm{t}}$ be the fixed point of $\zeta$ whose first letter is $(c A)$. Let $\phi: G \rightarrow\{c, 1,2\}$ be the letter-to-letter morphism given by

$$
\begin{aligned}
& \phi((c A))=c, \quad \phi((A B))=1, \quad \phi((B C))=2, \\
& \phi((C D))=1, \quad \phi((D A))=2, \quad \phi((B A))=2 .
\end{aligned}
$$

We have $\phi(\overline{\mathrm{t}})=c(12)^{\omega}=\mathrm{x}$.

Using Proposition 7 we obtain a slight improvement of the main results of respectively [Du2] and [Du3]. More precisely:

Theorem 8. Let $\alpha$ and $\beta$ be two multiplicatively independent Perron numbers. Let $\mathrm{x}$ be a sequence on a finite alphabet. The sequence $\mathrm{x}$ is both $\alpha$ substitutive primitive and $\beta$-substitutive primitive if and only if it is periodic.

Theorem 9. Let $U$ and $V$ be two Bertrand numeration systems, and let $\alpha$ and $\beta$ be multiplicatively independent $\beta$-numbers such that $L(U)=$ $L(\alpha)$ and $L(V)=L(\beta)$. Let $E$ be a subset of $\mathbb{N}$. The set $E$ is both $U$ recognizable and $V$-recognizable if and only if it is a finite union of arithmetic progressions. (See [Du3] for the terminology.)

4. Multiplicative independence and density. This section is devoted to the proof of the following proposition.

Proposition 10. Let $\alpha$ and $\beta$ be two rationally independent positive numbers (i.e., $\alpha / \beta \notin \mathbb{Q}$ ). Let $d$ and e be non-negative integers. Then the set

$$
\{n \alpha+d \log n-m \beta-e \log m ; n, m \in \mathbb{N}\}
$$

is dense in $\mathbb{R}$. 
The following straightforward corollary will be essential in the next section.

Corollary 11. Let $\alpha$ and $\beta$ be multiplicatively independent positive numbers. Let $d$ and $e$ be non-negative integers. Then the set

$$
\left\{\frac{n^{d} \alpha^{n}}{m^{e} \beta^{m}} ; n, m \in \mathbb{N}\right\}
$$

is dense in $\mathbb{R}^{+}$.

These two results are well known for $d=e=0$ (see [HW] for example). We need the following lemma to prove Proposition 10.

Lemma 12. Let $\beta<\alpha$ be two rationally independent numbers. Then for all $\varepsilon>0$ and all $N \in \mathbb{N}$ there exist $m, n$, with $m \geq n \geq N$, such that $0<n \alpha-m \beta<\varepsilon$.

Proof. The proof is left to the reader.

Proof of Proposition 10. Let $l \in \mathbb{R}$ and $\varepsilon>0$. We have to find $N, M \in \mathbb{N}$ such that $|N \alpha+d \log N-M \beta-e \log M-l|<\varepsilon$. The proof is divided into several cases.

CASE 1: $\alpha>\beta, e=d$ and $l \geq d \log (\beta / \alpha)$. From Lemma 12 there exist integers $0<n<m$ such that $0<n \alpha-m \beta<\varepsilon / 2$ and $d \log (1+\varepsilon /(m \beta)) \leq$ $\varepsilon / 2$. Hence

$$
d \log \left(\frac{\beta}{\alpha}\right)<d \log n-e \log m<d \log \left(\frac{\beta}{\alpha}\right)+d \log \left(1+\frac{\varepsilon}{m \beta}\right) .
$$

Then $n \alpha-m \beta+d(\log n-\log m)<l+\varepsilon$. Define $f: \mathbb{N} \rightarrow \mathbb{R}$ by

$$
f(k)=k(n \alpha-m \beta)-d(\log (k m)-\log (k n)) .
$$

We have $f(1)<l+\varepsilon, \lim _{k \rightarrow \infty} f(k)=\infty$ and $0<f(k+1)-f(k)=n \alpha-m \beta$ $<\varepsilon$. Hence there exists $k_{0} \in \mathbb{N}$ such that $\left|f\left(k_{0}\right)-l\right|<\varepsilon$, that is to say,

$$
|N \alpha+d \log N-M \beta-e \log M-l|<\varepsilon
$$

where $N=n k_{0}$ and $M=m k_{0}$.

CASE 2: $\alpha>\beta, e=d$ and $l<d \log (\beta / \alpha)$. It suffices to take $n, m$ with $0<n<m$ such that $-\varepsilon / 2<n \alpha-m \beta<0$ and $d \log (1+\varepsilon /(m \beta)) \leq \varepsilon / 2$, and the same method will give the result.

CASE 3: $\alpha>\beta, e>d$. Let $k_{0} \in \mathbb{N}$ be such that $-\varepsilon<(d-e) \log \left(1+1 / k_{0}\right)$ $<0$. If integers $n, m$ with $0<n<m$ are such that $0<n \alpha-m \beta<\varepsilon$ then

$$
\begin{aligned}
(d-e) \log m+d \log \left(\frac{\beta}{\alpha}\right) & <d \log n-e \log m \\
& <(d-e) \log m+d \log \left(\frac{\beta}{\alpha}\right)+d \log \left(1+\frac{\varepsilon}{m \beta}\right),
\end{aligned}
$$


which is negative for $m$ large enough. Hence from Lemma 12 it follows that there exist integers $n, m$ with $0<n<m$ such that $0<n \alpha-m \beta<\varepsilon$ and

$$
d \log n-e \log m \leq l-\left(k_{0}\right) \varepsilon-(d-e) \log k_{0} .
$$

Define $f: \mathbb{N} \rightarrow \mathbb{R}$ by

$$
f(k)=k(n \alpha-m \beta)+d \log (k n)-e \log (k m) .
$$

We have

$$
f\left(k_{0}\right) \leq k_{0} \varepsilon+(d-e) \log k_{0}+d \log n-e \log m \leq l .
$$

Moreover $\lim _{k \rightarrow \infty} f(k)=\infty$ and for all $k \geq k_{0}$,

$$
-\varepsilon<f(k+1)-f(k)=n \alpha-m \beta+(d-e) \log \left(1+\frac{1}{k}\right)<\varepsilon .
$$

Hence there exists an integer $k_{1} \geq k_{0}$ such that $\left|f\left(k_{1}\right)-l\right|<\varepsilon$, that is to say,

$$
|N \alpha+d \log N-M \beta-e \log M-l|<\varepsilon
$$

where $N=n k_{1}$ and $M=m k_{1}$.

REMAINING CASES: The same ideas complete the proof.

5. The letters appear with bounded gaps. Let $\alpha$ and $\beta$ be two multiplicatively independent Perron numbers. Let $\sigma$ and $\tau$ be substitutions on the alphabets $A$ and $B$, with fixed points $y$ and $z$ and with growth types $(d, \alpha)$ and $(e, \beta)$ respectively. Let $\phi: A \rightarrow C$ and $\psi: B \rightarrow C$ be letter-toletter morphisms such that $\phi(\mathrm{y})=\psi(\mathrm{z})=\mathrm{x}$. This section is devoted to the proof of the following proposition.

Proposition 13. The letters of $C$ which have infinitely many occurrences appear in $\mathrm{x}$ with bounded gaps in $\mathrm{x}$.

Proof. We prove this proposition considering two cases. Let $c \in C$ which has infinitely many occurrences. Let $X=\left\{n \in \mathbb{N} ; \mathbf{x}_{n}=c\right\}$ and $A^{\prime}=\{a \in A$; $\phi(a)=c\}$. Assume that the letter $c$ does not appear with bounded gaps. Then there exist $a \in A$ with infinitely many occurrences in y and a strictly increasing sequence $\left(p_{n} ; n \in \mathbb{N}\right)$ of positive integers such that the letter $c$ does not appear in $\phi\left(\sigma^{p_{n}}(a)\right)$. Let $A^{\prime \prime}$ be the set of such letters. We consider two cases.

CASE 1: There exists $a \in A^{\prime \prime}$ of maximal growth. Let $u \in A^{*}$ be such that $u a$ is a prefix of $\mathrm{y}$. Of course we can suppose that $u$ is non-empty. For all $n \in \mathbb{N}$ we call $\Omega_{n} \subset A$ the set of letters appearing in $\sigma^{p_{n}}(a)$. There exist distinct integers $n_{1}<n_{2}$ such that $\Omega_{n_{1}}=\Omega_{n_{2}}$. Let $\Omega$ be the set of letters appearing in $\sigma^{p_{n_{2}}-p_{n_{1}}}\left(\Omega_{n_{1}}\right)$. It is easy to show that $\Omega=\Omega_{n_{1}}=\Omega_{n_{2}}$. Consequently, the set of letters appearing in $\sigma^{p_{n_{2}}-p_{n_{1}}}(\Omega)$ is equal to $\Omega$ and for all $k \in \mathbb{N}$ the set of letters appearing in $\sigma^{p_{n_{1}}+k\left(p_{n_{2}}-p_{n_{1}}\right)}(A)$ is equal to 
$\Omega$. We set $p=p_{n_{1}}$ and $g=p_{n_{2}}-p_{n_{1}}$. We remark that the letter $c$ does not appear in the word $\phi\left(\sigma^{p+k g}(a)\right)$ and that $\left[\left|\sigma^{p+k g}(u)\right|,\left|\sigma^{p+k g}(u a)\right|[\cap X=\emptyset\right.$ for all $k \in \mathbb{N}$.

There exists a letter $a^{\prime}$ of maximal growth having an occurrence in $\sigma^{p}(a)$. We set $\sigma^{p}(a)=w a^{\prime} w^{\prime}$. For all $k \in \mathbb{N}$ we have $\left|\sigma^{p+k g}(u a)\right| \geq\left|\sigma^{k g}\left(\sigma^{p}(u) w a^{\prime}\right)\right|$ and

$$
\left[\left|\sigma^{k g}(v)\right|,\left|\sigma^{k g}\left(v w a^{\prime}\right)\right|[\cap X=\emptyset\right.
$$

where $v=\sigma^{p}(u)$. Because $a^{\prime}$ is of maximal growth we have $\lambda_{\sigma}(v)<\lambda_{\sigma}\left(v w a^{\prime}\right)$. Consequently, there exists an $\varepsilon>0$ such that

$$
\lambda_{\sigma}(v)(1+\varepsilon)<\lambda_{\sigma}\left(v w a^{\prime}\right)(1-\varepsilon) .
$$

From Lemma 5 we deduce that there exists $k_{0}$ such that for all $k \geq k_{0}$,

$$
\frac{\left|\sigma^{k g}(v)\right|}{(k g)^{d} \alpha^{k g}}<\lambda_{\sigma}(v)(1+\varepsilon)<\lambda_{\sigma}\left(v w a^{\prime}\right)(1-\varepsilon)<\frac{\left|\sigma^{k g}\left(v w a^{\prime}\right)\right|}{(k g)^{d} \alpha^{k g}} .
$$

From Lemma 6 applied to $\tau$ we find that there exist $s \in B^{*}$ of maximal growth, $t, t^{\prime} \in B^{*}$ and $h \in \mathbb{N}^{*}$ such that for all $n \in \mathbb{N}$,

$$
\psi\left(\mathrm{y}_{\left[\tau^{h n}(s) \tau^{h(n-1)}(t) \ldots \tau^{h}(t) t t^{\prime}\right]}\right)=c .
$$

From the second part of Lemma 6 it follows that there exists $\gamma \in \mathbb{R}$ such that

$$
\lim _{n \rightarrow \infty} \frac{\left|\tau^{h n}(s) \tau^{h(n-1)}(t) \ldots \tau^{h}(t) t t^{\prime}\right|}{(n h)^{e} \beta^{h n}}=\gamma .
$$

From Corollary 11 it comes that there exist two strictly increasing sequences of integers, $\left(m_{i} ; i \in \mathbb{N}\right)$ and $\left(n_{i} ; i \in \mathbb{N}\right)$, and $l \in \mathbb{R}$ such that

$$
\left.\frac{\gamma\left(m_{i} h\right)^{e} \beta^{m_{i} h}}{\left(n_{i} g\right)^{d} \alpha^{n_{i} g}} \underset{i \rightarrow \infty}{\longrightarrow} l \in\right] \lambda_{\sigma}(v)(1+\varepsilon), \lambda_{\sigma}\left(v w a^{\prime}\right)(1-\varepsilon)[.
$$

Hence from Lemma 5 we also have

$$
\begin{aligned}
& \frac{\left|\tau^{h m_{i}}(s) \tau^{h\left(m_{i}-1\right)}(t) \ldots \tau^{h}(t) t t^{\prime}\right|}{\left(n_{i} g\right)^{d} \alpha^{n_{i} g}} \\
& \quad=\frac{\left|\tau^{h m_{i}}(s) \tau^{h\left(m_{i}-1\right)}(t) \ldots \tau^{h}(t) t t^{\prime}\right|}{\gamma\left(m_{i} h\right)^{e} \beta^{m_{i} h}} \cdot \frac{\gamma\left(m_{i} h\right)^{e} \beta^{m_{i} h}}{\left(n_{i} g\right)^{d} \alpha^{n_{i} g}} \underset{i \rightarrow \infty}{\longrightarrow} l .
\end{aligned}
$$

From (4) and (5) there exists $i \in \mathbb{N}$ such that

$$
\left|\sigma^{n_{i} g}(v)\right|<\left|\tau^{h m_{i}}(s) \tau^{h\left(m_{i}-1\right)}(t) \ldots \tau^{h}(t) t t^{\prime}\right|<\left|\sigma^{n_{i} g}\left(v w a^{\prime}\right)\right|,
$$

which means that $\left|\tau^{h m_{i}}(s) \tau^{h\left(m_{i}-1\right)}(t) \ldots \tau^{h}(t) t t^{\prime}\right|$ belongs to $X$. This gives a contradiction with (3).

CASE 2: No letter in $A^{\prime \prime}$ has maximal growth. We define $B^{\prime \prime}$ as $A^{\prime \prime}$ but with respect to $\tau$ and $B$. We can suppose that no letter of $B^{\prime \prime}$ has maximal growth. There exists a letter $a \in A^{\prime \prime}$ (resp. $b \in B^{\prime \prime}$ ) which has infinitely many occurrences in y (resp. z) and with growth type $\left(d^{\prime}, \alpha^{\prime}\right)<(d, \alpha)$ (resp. 
$\left.\left(e^{\prime}, \beta^{\prime}\right)<(e, \beta)\right)$. We recall that $\alpha^{\prime}$ and $\beta^{\prime}$ are greater than 1 . Furthermore we can suppose that $\left(d^{\prime}, \alpha^{\prime}\right)$ (resp. $\left.\left(e^{\prime}, \beta^{\prime}\right)\right)$ is maximal with respect to $A^{\prime \prime}$ (resp. $\left.B^{\prime \prime}\right)$.

Let $w=w_{0} \ldots w_{n}$ be a word belonging to $L(\mathrm{y})($ resp. $L(\mathrm{z}))$. Denote by $\operatorname{gap}(w)$ the largest integer $k$ such that there exists $0 \leq i \leq n-k+1$ for which the letter $c$ does not appear in $\phi\left(w_{i} \ldots w_{i+k-1}\right)$ (resp. in $\psi\left(w_{i} \ldots w_{i+k-1}\right)$ ).

There exist infinitely many prefixes of y (resp. z) of the type $u_{1} a u_{2} a^{\prime}$ (resp. $v_{1} b v_{2} b^{\prime}$ ) fulfilling conditions (i) and (ii) below:

(i) The growth type of $u_{1} \in A^{*}$ and $a^{\prime} \in A$ (resp. $v_{1} \in B^{*}$ and $b^{\prime} \in B$ ) is maximal.

(ii) The words $u_{2}$ and $v_{2}$ do not contain a letter of maximal growth.

It is easy to prove that there exists a constant $K^{\prime}$ such that $\operatorname{gap}\left(\tau^{n}\left(b^{\prime}\right)\right) \leq$ $K^{\prime} n^{e^{\prime}} \beta^{\prime n}$ and $\operatorname{gap}\left(\sigma^{n}\left(a^{\prime}\right)\right) \leq K^{\prime} n^{d^{\prime}} \alpha^{\prime n}$ for all $n \in \mathbb{N}$. Due to Lemma 3, $\lim _{n \rightarrow \infty}\left|\sigma^{n}(a)\right| / n^{d^{\prime}} \alpha^{\prime n}$ and $\lim _{n \rightarrow \infty}\left|\tau^{n}(b)\right| / n^{e^{\prime}} \beta^{\prime n}$ exist and are finite; we call them $\mu(a)$ and $\mu(b)$ respectively.

Let $u_{1} a u_{2} a^{\prime}$ be a prefix of y fulfilling conditions (i) and (ii). Then choose $v_{1} b v_{2} b^{\prime}$ fulfilling the same conditions and so that

$$
\frac{K^{\prime}}{\mu(a)}\left(\frac{2 \lambda_{\sigma}\left(u_{1}\right)}{2 \lambda_{\tau}\left(v_{1}\right)+\lambda_{\tau}\left(b^{\prime}\right)}\right)^{\frac{\log \alpha^{\prime}}{\log \alpha}}\left(\frac{\log \beta}{\log \alpha}\right)^{e \frac{\log \beta^{\prime}}{\log \beta}-e^{\prime}} \leq \frac{1}{3} .
$$

From Corollary 11 there exist four strictly increasing sequences of integers $\left(m_{i} ; i \in \mathbb{N}\right),\left(n_{i} ; i \in \mathbb{N}\right),\left(p_{i} ; i \in \mathbb{N}\right)$ and $\left(q_{i} ; i \in \mathbb{N}\right)$ such that

(7) $\lim _{i \rightarrow \infty} \frac{n_{i}^{d} \alpha^{n_{i}}}{m_{i}^{e} \beta^{m_{i}}}=\frac{2 \lambda_{\tau}\left(v_{1}\right)}{2 \lambda_{\sigma}\left(u_{1}\right)+\lambda_{\sigma}\left(a^{\prime}\right)}, \quad \lim _{i \rightarrow \infty} \frac{p_{i}^{e} \beta^{p_{i}}}{q_{i}^{d} \alpha^{q_{i}}}=\frac{2 \lambda_{\sigma}\left(u_{1}\right)}{2 \lambda_{\tau}\left(v_{1}\right)+\lambda_{\tau}\left(b^{\prime}\right)}$.

As a consequence of (7) we have

$$
\lim _{i \rightarrow \infty} n_{i} / m_{i}=\log \beta / \log \alpha \quad \text { and } \quad \lim _{i \rightarrow \infty} p_{i} / q_{i}=\log \alpha / \log \beta,
$$

and there exists $i_{0}$ such that for all $i \geq i_{0}$ we have

$$
\frac{\left|\sigma^{n_{i}}\left(u_{1} a u_{2}\right)\right|}{\left|\tau^{m_{i}}\left(v_{1}\right)\right|} \leq 1 \leq \frac{\left|\sigma^{n_{i}}\left(u_{1} a u_{2} a^{\prime}\right)\right|}{\left|\tau^{m_{i}}\left(v_{1} b\right)\right|}, \quad \frac{\left|\tau^{p_{i}}\left(v_{1} b v_{2}\right)\right|}{\left|\sigma^{q_{i}}\left(u_{1}\right)\right|} \leq 1 \leq \frac{\left|\tau^{p_{i}}\left(v_{1} b v_{2} b^{\prime}\right)\right|}{\left|\sigma^{q_{i}}\left(u_{1} a\right)\right|} .
$$

It follows that $\psi\left(\tau^{m_{i}}(b)\right)$ (resp. $\left.\phi\left(\sigma^{q_{i}}(a)\right)\right)$ has an occurrence in $\phi\left(\sigma^{n_{i}}\left(a^{\prime}\right)\right)$ (resp. $\left.\psi\left(\tau^{p_{i}}\left(b^{\prime}\right)\right)\right)$.

To obtain a contradiction it suffices to prove that there exists $j \geq i_{0}$ such that

$$
\operatorname{gap}\left(\sigma^{n_{j}}\left(a^{\prime}\right)\right) /\left|\tau^{m_{j}}(b)\right| \leq 1 / 2 \quad \text { or } \quad \operatorname{gap}\left(\tau^{p_{j}}\left(b^{\prime}\right)\right) /\left|\sigma^{q_{j}}(a)\right| \leq 1 / 2 .
$$

We will consider several cases. First we define $K$ to be the maximum of the set

$$
\left\{K^{\prime}, 2 \frac{\log \beta}{\log \alpha}, 2 \frac{\log \alpha}{\log \beta}, \frac{4 \lambda_{\tau}\left(v_{1}\right)}{2 \lambda_{\sigma}\left(u_{1}\right)+\lambda_{\sigma}\left(a^{\prime}\right)}, \frac{4 \lambda_{\sigma}\left(u_{1}\right)}{2 \lambda_{\tau}\left(v_{1}\right)+\lambda_{\tau}\left(b^{\prime}\right)}\right\} .
$$


We remark that $K \geq 2$. There exists $j_{0}$ such that for all $i \geq j_{0}$ the quantities

$$
\frac{n_{i}}{m_{i}}, \frac{p_{i}}{q_{i}}, \frac{n_{i}^{d} \alpha^{n_{i}}}{m_{i}^{e} \beta^{m_{i}}}, \frac{p_{i}^{e} \beta^{p_{i}}}{q_{i}^{d} \alpha^{q_{i}}}, \frac{\mu(a) q_{i}^{d^{\prime}} \alpha^{\prime q_{i}}}{\left|\sigma^{q_{i}}(a)\right|}, \frac{\mu(b) m_{i}^{e^{\prime}} \beta^{\prime m_{i}}}{\left|\tau^{m_{i}}(b)\right|}, \frac{\operatorname{gap}\left(\sigma^{n_{i}}\left(a^{\prime}\right)\right)}{n_{i}^{d^{\prime}} \alpha^{\prime n_{i}}}
$$

are less than $K$. Let $i \geq j_{0}$. To find $j$ we will consider five cases.

CASE 2.1: $\log \alpha / \log \beta<\log \alpha^{\prime} / \log \beta^{\prime}$. As $\beta^{\prime}>1$ we have

$$
\begin{aligned}
\operatorname{gap}\left(\tau^{p_{i}}\left(b^{\prime}\right)\right) /\left|\sigma^{q_{i}}(a)\right| & \leq \frac{K p_{i}^{e^{\prime}} \beta^{p_{i}}}{\mu(a) q_{i}^{d^{\prime}} \alpha^{\prime q_{i}}} \cdot \frac{\mu(a) q_{i}^{d^{\prime}} \alpha^{\prime q_{i}}}{\left|\sigma^{q_{i}}(a)\right|} \\
& \leq \frac{K^{2}}{\mu(a)} \cdot \frac{p_{i}^{e^{\prime}}}{q_{i}^{d^{\prime}}} \exp \left\{\left(\frac{p_{i}}{q_{i}}-\frac{\log \alpha^{\prime}}{\log \beta^{\prime}}\right) q_{i} \log \beta^{\prime}\right\},
\end{aligned}
$$

which tends to 0 as $i$ tends to $\infty$ (this follows from (8)).

CASE 2.2: $\log \alpha^{\prime} / \log \beta^{\prime}<\log \alpha / \log \beta$. As in the first case we obtain

$$
\lim _{i \rightarrow \infty} \operatorname{gap}\left(\sigma^{n_{i}}\left(a^{\prime}\right)\right) /\left|\tau^{m_{i}}(b)\right|=0 .
$$

CASE 2.3: $\log \alpha^{\prime} / \log \alpha=\log \beta^{\prime} / \log \beta$ and $\left(e^{\prime}-d^{\prime}\right) \log \beta<(e-d) \log \beta^{\prime}$. We have

$$
\begin{aligned}
\operatorname{gap}\left(\tau^{p_{i}}\left(b^{\prime}\right)\right) /\left|\sigma^{q_{i}}(a)\right| & \leq \frac{K^{2}}{\mu(a)} \cdot \frac{p_{i}^{e^{\prime}}}{q_{i}^{d^{\prime}}} \cdot \frac{\beta^{\prime p_{i}}}{\alpha^{\prime q_{i}}}=\frac{K^{2}}{\mu(a)} \cdot \frac{p_{i}^{e^{\prime}}}{q_{i}^{d^{\prime}}}\left(\frac{\beta^{p_{i}}}{\alpha^{q_{i}}}\right)^{\frac{\log \beta^{\prime}}{\log \beta}} \\
& =\frac{K^{2}}{\mu(a)} \cdot \frac{p_{i}^{e^{\prime}}}{q_{i}^{d^{\prime}}}\left(\frac{q_{i}^{d}}{p_{i}^{e}}\right)^{\frac{\log \beta^{\prime}}{\log \beta}}\left(\frac{p_{i}^{e} \beta^{p_{i}}}{q_{i}^{d} \alpha^{q_{i}}}\right)^{\frac{\log \beta^{\prime}}{\log \beta}} \\
& \leq \frac{K^{2}}{\mu(a)}\left(\frac{p_{i}}{q_{i}}\right)^{e^{\prime}-\frac{\log \beta^{\prime}}{\log \beta}} K^{\frac{\log \beta^{\prime}}{\log \beta}} q_{i}^{\left(e^{\prime}-d^{\prime}\right)-(e-d) \frac{\log \beta^{\prime}}{\log \beta}} \\
& \leq \frac{K^{2}}{\mu(a)} K^{e^{\prime}+(1-e) \frac{\log \beta^{\prime}}{\log \beta}} q_{i}^{\left(e^{\prime}-d^{\prime}\right)-(e-d) \frac{\log \beta^{\prime}}{\log \beta}},
\end{aligned}
$$

which tends to 0 as $i$ tends to $\infty$.

CASE 2.4: $\log \alpha^{\prime} / \log \alpha=\log \beta^{\prime} / \log \beta$ and $\left(e^{\prime}-d^{\prime}\right) \log \beta>(e-d) \log \beta^{\prime}$. As in the previous case we obtain

$$
\lim _{i \rightarrow \infty} \operatorname{gap}\left(\sigma^{n_{i}}\left(a^{\prime}\right)\right) /\left|\tau^{m_{i}}(b)\right|=0 .
$$

CASE 2.5: $\log \alpha^{\prime} / \log \alpha=\log \beta^{\prime} / \log \beta$ and $\left(e^{\prime}-d^{\prime}\right) \log \beta=(e-d) \log \beta^{\prime}$. From (6)-(8) we obtain, for all large enough $i$,

$$
\begin{aligned}
\operatorname{gap}\left(\tau^{p_{i}}\left(b^{\prime}\right)\right) /\left|\sigma^{q_{i}}(a)\right| & \leq \frac{K^{\prime}}{\mu(a)} \cdot \frac{p_{i}^{e^{\prime}} \beta^{\prime p_{i}}}{q_{i}^{d^{\prime}} \alpha^{\prime q_{i}}} \cdot \frac{\mu(a) q_{i}^{d^{\prime}} \alpha^{\prime q_{i}}}{\left|\sigma^{q_{i}}(a)\right|} \\
& \leq \frac{K^{\prime}}{\mu(a)}\left(\frac{p_{i}^{e} \beta^{p_{i}}}{q_{i}^{d} \alpha^{q_{i}}}\right)^{\frac{\log \alpha^{\prime}}{\log \alpha}}\left(\frac{q_{i}}{p_{i}}\right)^{e^{\frac{\log \beta^{\prime}}{\log \beta}-e^{\prime}}} \frac{\mu(a) q_{i}^{d^{\prime}} \alpha^{\prime q_{i}}}{\left|\sigma^{q_{i}}(a)\right|} \leq \frac{1}{2}
\end{aligned}
$$


COROLLARY 14. The words having infinitely many occurrences in $\times$ appear in $\times$ with bounded gaps.

Proof. Let $u$ be such a word. We set $|u|=n$. It suffices to prove that the letter 1 appears with bounded gaps in the sequence $t \in\{0,1\}^{\mathbb{N}}$ defined by

$$
\mathrm{t}_{i}=1 \quad \text { if } \mathrm{x}_{[i, i+n-1]}=u
$$

and 0 otherwise. Below we prove that $\mathrm{t}$ is $\alpha$ and $\beta$-substitutive.

The sequence $\mathrm{y}^{(n)}=\left(\left(\mathrm{y}_{i} \ldots \mathrm{y}_{i+n-1}\right) ; i \in \mathbb{N}\right)$ is a fixed point of the substitution $\sigma_{n}: A_{n} \rightarrow A_{n}^{*}$ where $A_{n}$ is the alphabet $A^{n}$, defined for all $\left(a_{1} \ldots a_{n}\right)$ in $A_{n}$ by

$$
\sigma_{n}\left(\left(a_{1} \ldots a_{n}\right)\right)=\left(b_{1} \ldots b_{n}\right)\left(b_{2} \ldots b_{n+1}\right) \ldots\left(b_{\left|\sigma\left(a_{1}\right)\right|} \ldots b_{\left|\sigma\left(a_{1}\right)\right|+n-1}\right)
$$

where $\sigma\left(a_{1} \ldots a_{n}\right)=b_{1} \ldots b_{k}$ (for more details see Section V.4 of [Qu] for example).

Let $\varrho: A_{n} \rightarrow A^{*}$ be the letter-to-letter morphism defined by $\varrho\left(\left(b_{1} \ldots b_{n}\right)\right)$ $=b_{1}$ for all $\left(b_{1} \ldots b_{n}\right) \in A_{n}$. We have $\varrho \circ \sigma_{n}=\sigma \circ \varrho$, and then $M_{\varrho} M_{\sigma_{n}}=$ $M_{\sigma} M_{\varrho}$. Consequently, the dominant eigenvalue of $\sigma_{n}$ is $\alpha$ and $\mathrm{y}^{(n)}$ is $\alpha$ substitutive. Let $f: A_{n} \rightarrow\{0,1\}$ be the letter-to-letter morphism defined by

$$
f\left(\left(b_{1} \ldots b_{n}\right)\right)=1 \quad \text { if } b_{1} \ldots b_{n}=u \text { and } 0 \text { otherwise. }
$$

It is easy to see that $f\left(\mathrm{y}^{(n)}\right)=\mathrm{t}$ hence $\mathrm{t}$ is $\alpha$-substitutive. In the same way we show that $\mathrm{t}$ is $\beta$-substitutive and Theorem 13 concludes the proof.

\section{Proof of Theorem 1}

6.1. Decomposition of a substitution into sub-substitutions. The following proposition is a consequence of Section 4.4 and Proposition 4.5.6 of $[\mathrm{LM}]$.

Proposition 15. Let $M=\left(m_{i, j}\right)_{i, j \in A}$ be a matrix with non-negative coefficients and no zero column. There exist positive integers $p \neq 0, q, l$, where $q \leq l-1$, and a partition $\left\{A_{i} ; 1 \leq i \leq l\right\}$ of $A$ such that the matrix $M^{p}$ is equal to

$\left.\begin{array}{l|cccccccc} & A_{1} & A_{2} & \ldots & A_{q} & A_{q+1} & A_{q+2} & \ldots & A_{l} \\ A_{1} & M_{1} & 0 & \ldots & 0 & 0 & 0 & \ldots & 0 \\ A_{2} & M_{1,2} & M_{2} & \ldots & 0 & 0 & 0 & \ldots & 0 \\ \vdots & \vdots & \vdots & \ddots & \vdots & \vdots & \vdots & \vdots & \vdots \\ A_{q} & M_{1, q} & M_{2, q} & \ldots & M_{q} & 0 & 0 & \ldots & 0 \\ A_{q+1} & M_{1, q+1} & M_{2, q+1} & \ldots & M_{q, q+1} & M_{q+1} & 0 & \ldots & 0 \\ A_{q+2} & M_{1, q+2} & M_{2, q+2} & \ldots & M_{q, q+2} & 0 & M_{q+2} & \ldots & 0 \\ \vdots & \vdots & \vdots & \ddots & \vdots & \vdots & \vdots & \ddots & \vdots \\ A_{l} & M_{1, l} & M_{2, l} & \ldots & M_{q, l} & 0 & 0 & \ldots & M_{l}\end{array}\right)$


where the matrices $M_{i}, 1 \leq i \leq q$ (resp. $q+1 \leq i \leq l$ ), are primitive or zero (resp. primitive), and such that for all $1 \leq i \leq q$ there exists $i+1 \leq j \leq l$ such that the matrix $M_{i, j}$ is different from 0.

In what follows we keep the notations of Proposition 15. We will say that $\left\{A_{i} ; 1 \leq i \leq l\right\}$ is a primitive component partition of $A$ (with respect to $M)$. If $i$ belongs to $\{q+1, \ldots, l\}$ we will say that $A_{i}$ is a principal primitive component of $A$ (with respect to $M$ ).

Let $\tau: A \rightarrow A^{*}$ be a substitution and $M=\left(m_{i, j}\right)_{i, j \in A}$ its matrix. Let $i \in\{q+1, \ldots, l\}$. We denote by $\tau_{i}$ the restriction $\left(\tau^{p}\right)_{\mid A_{i}}: A_{i} \rightarrow A^{*}$ of $\tau^{p}$ to $A_{i}$. Because $\tau_{i}\left(A_{i}\right)$ is included in $A_{i}^{*}$ we can view $\tau_{i}$ as a morphism from $A_{i}$ to $A_{i}^{*}$ with matrix $M_{i}$. Let $i \in\{1, \ldots, q\}$ be such that $M_{i}$ is not equal to 0 . Let $\varphi_{i}$ be the morphism from $A$ to $A_{i}^{*}$ defined by $\varphi(b)=b$ if $b$ belongs to $A_{i}$ and the empty word otherwise. Consider the map $\tau_{i}: A_{i} \rightarrow A^{*}$ defined by $\tau_{i}(b)=\varphi_{i}\left(\tau^{p}(b)\right)$ for all $a \in A_{i}$. We remark as previously that $\tau_{i}\left(A_{i}\right)$ is included in $A_{i}^{*}$, and consequently $\tau_{i}$ defines a morphism from $A_{i}$ to $A_{i}^{*}$ with matrix $M_{i}$.

We will say that the substitution $\tau: A \rightarrow A^{*}$ satisfies Condition (C) if:

C1. The matrix $M$ is of the type (9) (i.e., $p=1$ );

$\mathrm{C} 2$. The matrices $M_{i}$ are 0 or have positive entries if $1 \leq i \leq q$, and have positive entries otherwise;

C3. For all matrices $M_{i}$ different from 0 , with $i \in\{1, \ldots, l\}$, there exists $a_{i} \in A_{i}$ such that $\tau_{i}\left(a_{i}\right)=a_{i} u_{i}$ where $u_{i}$ is a non-empty word of $A^{*}$ if $M_{i}$ is different from the $1 \times 1$ matrix [1] and empty otherwise.

From Proposition 15 every substitution $\tau: A \rightarrow A^{*}$ has a power $\tau^{k}$ satisfying condition (C). The definition of substitutions implies that $M_{i} \neq[1]$ for all $q+1 \leq i \leq l$.

Let $\tau: A \rightarrow A^{*}$ be a substitution satisfying condition (C) (we keep the previous notations). For all $1 \leq i \leq l$ such that $M_{i}$ is different from 0 and from the $1 \times 1$ matrix [1], the map $\tau_{i}: A_{i} \rightarrow A_{i}^{*}$ defines a substitution which we will call a main sub-substitution of $\tau$ if $i \in\{q+1, \ldots, l\}$ and a non-main sub-substitution of $\tau$ otherwise. Moreover the matrix $M_{i}$ has positive coefficients, which implies that the substitution $\tau_{i}$ is primitive. We remark that there exists at least one main sub-substitution.

In [Du3] the following results were obtained:

Lemma 16. Let $\mathrm{x}$ be a proper fixed point of the substitution $\sigma$. Let $\bar{\sigma}$ : $\bar{A} \rightarrow \bar{A}^{*}$ be a main sub-substitution of $\sigma$. Then for all $n \in \mathbb{N}$ and all $a \in A$ the word $\sigma^{n}(a)$ appears infinitely many times in $\mathrm{x}$.

THEOREM 17. Let $\mathrm{x}$ and $\mathrm{y}$ be respectively a primitive $\alpha$-substitutive sequence and a primitive $\beta$-substitutive sequence such that $L(\mathrm{x})=L(\mathrm{y})$. Suppose that $\alpha$ and $\beta$ are multiplicatively independent. Then $\mathrm{x}$ and $\mathrm{y}$ are periodic. 
6.2. The conjecture for "good" substitutions. We have not yet succeeded in proving the conjecture given in the introduction but we are able to prove it for a very large family of substitutions. We call them "good" substitutions. More precisely, let $\sigma: A \rightarrow A^{*}$ be a substitution whose dominant eigenvalue is $\alpha$. The substitution $\sigma$ is said to be a "good" substitution if there exists a main sub-substitution whose dominant eigenvalue is $\alpha$. For example primitive substitutions and substitutions of constant length are "good" substitutions.

Now consider the following substitution.

$$
\sigma:\{a, 0,1\} \rightarrow\{a, 0,1\}^{*}, \quad a \mapsto a a 0,0 \mapsto 01,1 \mapsto 0 .
$$

Its dominant eigenvalue is 2 and it has only one main sub-substitution $(0 \mapsto$ $01,1 \mapsto 0)$ whose dominant eigenvalue is $(1+\sqrt{5}) / 2$, hence it is not a "good" substitution.

THEOREM 18. Suppose that we only consider "good" substitutions. Then the conjecture is true.

Proof. We adopt the notations of the first lines of Section 5. Let $\bar{\sigma}: \bar{A} \rightarrow$ $\bar{A}^{*}$ be a main sub-substitution of $\sigma$. The words of x appearing infinitely many times in $x$ appear with bounded gaps (Corollary 14). Hence using Lemma 16 we deduce that for all main sub-substitutions $\bar{\sigma}$ of $\sigma$ and $\bar{\tau}$ of $\tau$ we have $\phi(L(\bar{\sigma}))=\psi(L(\bar{\tau}))=L$. From Theorem 17 it follows that $L$ is periodic, i.e., there exists a word $u$ such that $L=L\left(u^{\omega}\right)$ where $|u|$ is the least period.

The word $u$ appears infinitely many times, hence it appears with bounded gaps. Let $\mathcal{R}_{u}$ be the set of return words to $u$ (a word $w$ is a return word to $u$ if $w u \in L(\mathrm{x}), u$ is a prefix of $w u$ and $u$ has exactly two occurrences in $w u$ ). This set is finite. There exists an integer $N$ such that all the words $w \in \mathcal{R}_{u} \cap L\left(\mathrm{x}_{N} \mathrm{x}_{N+1} \ldots\right)$ appear infinitely many times in $\mathrm{x}$. Hence these words appear with bounded gaps in $x$.

We can suppose that $u$ is a prefix of $\mathrm{t}=\mathrm{x}_{N} \mathrm{x}_{N+1} \ldots$ Then $\mathrm{t}$ is a concatenation of return words to $u$. Let $w$ be such a word. It appears with bounded gaps hence it appears in some $\phi\left(\bar{\sigma}^{n}(a)\right)$ and there exist two words, $p$ and $q$, and an integer $i$ such that $w u=p u^{i} q$. As $|u|$ is the least period of $L$ it follows that $w u=u^{i}$. Hence $\mathrm{t}=u^{\omega}$.

The case of fixed points. This part is devoted to the proof of Theorem 1 restricted to fixed points. More precisely we prove:

Corollary 19. Let $\mathrm{x}$ be a fixed point of the substitution $\sigma: A \rightarrow A^{*}$ whose dominant eigenvalue is $\alpha$. Suppose that $\mathrm{x}$ is also a fixed point of the substitution $\tau: A \rightarrow A^{*}$ whose dominant eigenvalue is $\beta$. Suppose that $\alpha$ and $\beta$ are multiplicatively independent. Then $\mathrm{x}$ is ultimately periodic.

Proof. The letters appearing infinitely often in $x$ appear with bounded 
gaps (Proposition 13). Let $\bar{\sigma}: \bar{A} \rightarrow \bar{A}^{*}$ be a main sub-substitution of $\sigma$. Let $a \in \bar{A}$. Suppose that there exists a letter $b$, appearing infinitely many times in $\mathrm{x}$, which does not belong to $\bar{A}$. Then the word $\sigma^{n}(a)$ does not contain $b$ and $b$ could not appear with bounded gaps. Consequently, there exists only one main sub-substitution and the letters which appear with bounded gaps belong to $\bar{A}$. Hence $\sigma$ is a "good" substitution. In the same way $\tau$ is a good substitution. Theorem 18 concludes the proof.

\section{References}

[BHMV1] V. Bruyère, G. Hansel, C. Michaux and R. Villemaire, Logic and p-recognizable sets of integers, Bull. Belg. Math. Soc. Simon Stevin 1 (1994), 191-238.

[BHMV2] - - - - - - Correction to: "Logic and p-recognizable sets of integers", ibid. 577 .

[Co1] A. Cobham, On the base-dependence of sets of numbers recognizable by finite automata, Math. Systems Theory 3 (1969), 186-192.

[Co2] - Uniform tag sequences, ibid. 6 (1972), 164-192.

[Du1] F. Durand, A characterization of substitutive sequences using return words, Discrete Math. 179 (1998), 89-101.

[Du2] - A generalization of Cobham's theorem, Theory Comput. Syst. 31 (1998), 169-185.

[Du3] - Sur les ensembles d'entiers reconnaissables, J. Théor. Nombres Bordeaux 10 (1998), 65-84.

[Ei] S. Eilenberg, Automata, Languages and Machines, Vol. A, Academic Press, 1974.

[Fa] S. Fabre, Une généralisation du théorème de Cobham, Acta Arith. 67 (1994), 197-208.

[Ha] G. Hansel, Systèmes de numération indépendants et syndéticité, Theoret. Comput. Sci. 204 (1998), 119-130.

[HW] G. H. Hardy and E. M. Wright, An Introduction to the Theory of Numbers, 5th ed., Oxford Univ. Press, 1979.

[LM] D. Lind and B. Marcus, An Introduction to Symbolic Dynamics and Coding, Cambridge Univ. Press, 1995.

[Qu] M. Queffélec, Substitution Dynamical Systems. Spectral Analysis, Lecture Notes in Math. 1294, Springer, Berlin, 1987.

[SS] A. Salomaa and M. Soittola, Automata-theoretic aspects of formal power series, Texts Monographs Comput. Sci., Springer, 1978.

Laboratoire Amiénois de Mathématiques Fondamentales et Appliquées

CNRS FRE 2270

Université de Picardie Jules Verne

33 rue Saint Leu

80039 Amiens Cedex 01, France

E-mail: fabien.durand@u-picardie.fr

Received on 23.10.2000

and in revised form on 6.12.2001 